\title{
Emerging Role of (Endo)Cannabinoids in Migraine
}

\author{
Pinja Leimuranta ${ }^{1}$, Leonard Khiroug ${ }^{2,3 *}$ and Rashid Giniatullin ${ }^{1,4 *}$ \\ ' A.I. Virtanen Institute for Molecular Sciences, University of Eastern Finland, Kuopio, Finland, ${ }^{2}$ Neurotar Ltd., Helsinki, \\ Finland, ${ }^{3}$ Neuroscience Center, University of Helsinki, Helsinki, Finland, ${ }^{4}$ Laboratory of Neurobiology, Kazan Federal \\ University, Kazan, Russia
}

In this mini-review, we summarize recent discoveries and present new hypotheses on the role of cannabinoids in controlling trigeminal nociceptive system underlying migraine pain. Individual sections of this review cover key aspects of this topic, such as: (i) the current knowledge on the endocannabinoid system (ECS) with emphasis on expression of its components in migraine related structures; (ii) distinguishing peripheral from central site of action of cannabinoids, (iii) proposed mechanisms of migraine pain and control of nociceptive traffic by cannabinoids at the level of meninges and in brainstem, (iv) therapeutic targeting in migraine of monoacylglycerol lipase and fatty acid amide hydrolase, enzymes which control the level of endocannabinoids; (v) dual (possibly opposing) actions of cannabinoids via anti-nociceptive CB1 and CB2 and

\section{OPEN ACCESS}

Edited by:

Fabricio A. Pamplona,

Entourage Phytolab, Brazil

Reviewed by:

Dasiel Oscar Borroto-Escuela, Karolinska Institute (KI), Sweden

Anna Maria Pittaluga,

Università di Genova, Italy

${ }^{*}$ Correspondence:

Rashid Giniatullin

Rashid.giniatullin@uef.fi Leonard Khiroug leonard.khirug@helsinki.fi

Specialty section: This article was submitted to

Neuropharmacology,

a section of the journal

Frontiers in Pharmacology

Received: 24 January 2018 Accepted: 10 April 2018 Published: 24 April 2018

Citation:

Leimuranta $P$, Khiroug $L$ and Giniatullin R (2018) Emerging Role of (Endo)Cannabinoids in Migraine.

Front. Pharmacol. 9:420.

doi: 10.3389/fphar.2018.00420 pro-nociceptive TRPV1 receptors. We explore the cannabinoid-mediated mechanisms in the frame of the Clinical Endocannabinoid Deficiency (CECD) hypothesis, which implies reduced tone of endocannabinoids in migraine patients. We further discuss the control of cortical excitability by cannabinoids via inhibition of cortical spreading depression (CSD) underlying the migraine aura. Finally, we present our view on perspectives of Cannabis-derived (extracted or synthetized marijuana components) or novel endocannabinoid therapeutics in migraine treatment.

Keywords: migraine, cannabinoids, CGRP, nociception, marijuana, cannabinoid receptor, TRPV1

\section{INTRODUCTION}

Migraine is a debilitating disorder most commonly characterized by a unilateral hemicranial pulsating headache often accompanied by a great variety of other symptoms such as sensory disturbances and nausea (Pavlovic et al., 2014; Russo, 2016). The full list of migraine criteria is provided in the latest version of Headache Classification (ICHD-3 beta, 2013). Due to its high prevalence and disruptive nature, the mechanisms contributing to migraine headache have been intensely studied over many decades but remain debatable. The current consensus states that migraine pain is caused by lowering of the threshold of nociceptive signal processing in response to release of pro-inflammatory agents. Migraine attack's initiation has been linked to both environmental and hormonal triggers (Pavlovic et al., 2014), which lead to pathophysiological changes due to a sterile neurogenic inflammation in meninges and activation of trigeminal sensory nerves (Pietrobon and Moskowitz, 2013; Gouveia-Figueira et al., 2017).

The multifaceted nature of migraine makes it difficult to define the exact criteria for clinical assessment, and may underlie the vast variability in the ways in which migraine patients respond to existing modes of treatment. Additionally, many of the anti-migraine therapies carry 
adverse effects, a challenge which has caused discontinuation of research and development of potential anti-migraine drugs (Russo, 2015). For these reasons, introduction of new, more inclusive and effective modes of therapy is urgently needed.

Different parts of Cannabis sativa plant have been utilized for centuries in treatment of multitude of health conditions, and consumption of this plant is often associated with psychotropic effects such as mood fluctuations, intoxication, euphoria, increased heart rate, physical dependence upon long-term use, and cognitive impairment (Niyuhire et al., 2007). Regarding migraine pathology, the vital characteristics justifying the proposed use of medical cannabis include anticonvulsive (Rosenberg et al., 2015), analgesic, antiemetic (Parker et al., 2011), and anti-inflammatory effects (Nagarkatti et al., 2009). Mainly due to their potent analgesic action, marijuana-derived exogenous cannabinoids are currently being used for symptomatic and prophylactic treatment in many pain conditions (Oláh et al., 2017), including migraineassociated pain (Chakrabarti et al., 2015). The use of exogenous cannabinoids has been greatly debated as a mode of therapy during past years, but the recent changes in legislation have facilitated their use in several countries. Following the push by the public for increasing cannabinoid availability, the demand for research on cannabinoid substances has also escalated.

This review aims to take a look at the recent publications on the effectiveness and safety of cannabinoid-based migraine treatment, as well as studies of the mechanisms underlying therapeutic effects of these compounds. Based on our experience in experimental studies of migraine, we discuss our own and other available data on the potential applications of cannabinoid therapy in migraine treatment.

\section{ENDOCANNABINOID SYSTEM: EXOGENOUS AND ENDOGENOUS AGONISTS}

Endocannabinoid system (ECS) is a comprehensive signaling system present in virtually every cell type and playing a critical role in maintaining body homoeostasis (Aizpurua-Olaizola et al., 2017). ECS' numerous components include the enzymes responsible for synthesis of endocannabinoids (eCBs), specific receptors of eCBs, and the post-activity neutralizing pathways (Marco et al., 2012). Here we provide only a short overview of this complex system related to discussion of migraine pathology.

To date, several major and many less explored components of the ECS have been identified (Chakrabarti et al., 2015). The most prevalent eCBs are 2-arachidonoylglycerol (2-AG) and arachidonoylethanolamine (anandamide, AEA) (Figure 1). Overall, 2-AG is considered the primary signaling molecule and is abundantly expressed throughout the brain (Sugiura et al., 2002). The action of eCBs is mimicked by the main pharmacological components of marijuana, namely phytocannabinoids ( $\mathrm{pCBs}$ ), including the psychotropic $\Delta 9$-tetrahydrocannabinol (THC) and the non-psychotropic cannabidiol (CBD) (Oláh et al., 2017; Figure 1).
The ECS signals are relayed primarily by two receptors: type 1 cannabinoid receptor (CB1), which is one of the most abundant G-protein coupled receptor in the brain (Smith et al., 2017), and type 2 cannabinoid receptor (CB2), which is functionally related to $\mathrm{CB} 1$ but is expressed primarily in peripheral tissues (Chakrabarti et al., 2015). Both CB1 and CB2 are natively activated by eCBs 2-AG and AEA, but they also respond to binding of pCBs. Thus, THC is thought to act primarily via its potent activation of $\mathrm{CB} 1$ and $\mathrm{CB} 2$. The mechanism of action is less clear for $\mathrm{CBD}$, which has been reported to affect more than 65 discrete molecular targets and to have varied effects outside of ECS (Bih et al., 2015).

One important issue remaining unsolved is how exactly eCBs are released from cells. The traditional dogma states that bioactive eCBs, unlike other neurotransmitters such as acetylcholine and dopamine, are produced "on-demand" (Marsicano et al., 2003). An alternative view suggests that eCBs may be pre-synthesized and stored, much like neurotransmitters (Maccarrone et al., 2010; Fonseca et al., 2013; Chakrabarti et al., 2015).

Endocannabinoid system is active in stress-responsive parts of central and peripheral nervous system, functioning to reduce pain and to alleviate neurodegenerative and inflammatory damage (Preedy, 2017; Smith et al., 2017). Short-term effects induced by eCBs have been shown to involve plastic changes in many brain areas affecting pain sensation (Oláh et al., 2017). All these mechanisms are linked, directly or indirectly, to the migraine pathology.

\section{MAPPING ECS EFFECTS IN MIGRAINE MODELS - CENTRAL VS. PERIPHERAL}

The importance of the trigeminovascular system (TGVS) in migraine pathophysiology is widely recognized by experts in the field. During a migraine attack, prolonged activation of the TGVS - comprising meningeal trigeminal nerves and vessels along with dural mast cells (MC) (Figure 1) - ultimately causes sensitization of higher order neurons in the central nervous system (CNS), leading to the persistent nociceptive signaling (Burstein et al., 2015). Furthermore, the resulting sensitization has been found to stimulate TGVS activity, creating a positive feedback loop (Eroli et al., 2017). The main migraine mediator associated with the TGVS system is the neuropeptide calcitonin gene-related peptide (CGRP), which promotes vasodilation and contributes to the sterile meningeal inflammation associated with sensitization of nociceptive pathway (Giniatullin et al., 2008; Villalón and Olesen, 2009; Pietrobon and Moskowitz, 2013; Dux et al., 2016; Figure 1). All three key meningeal structures (nerves, vessels and $\mathrm{MC}$ ) can act as targets for the action of pCBs or eCBs. Several papers from P. Goadsby lab have shown that CGRP-induced dilation of dural blood vessels and neuronal pro-nociceptive activity could be reduced by AEA (Akerman et al., 2003, 2007). MC, populating the TGVS in large quantities and responding to CGRP with degranulation (Figure 1), likely play a triggering role in migraine (Levy, 2010; Kilinc et al., 2017). In particular serotonin, a major component of mast cell granules, is able to produce a robust activation of trigeminal 


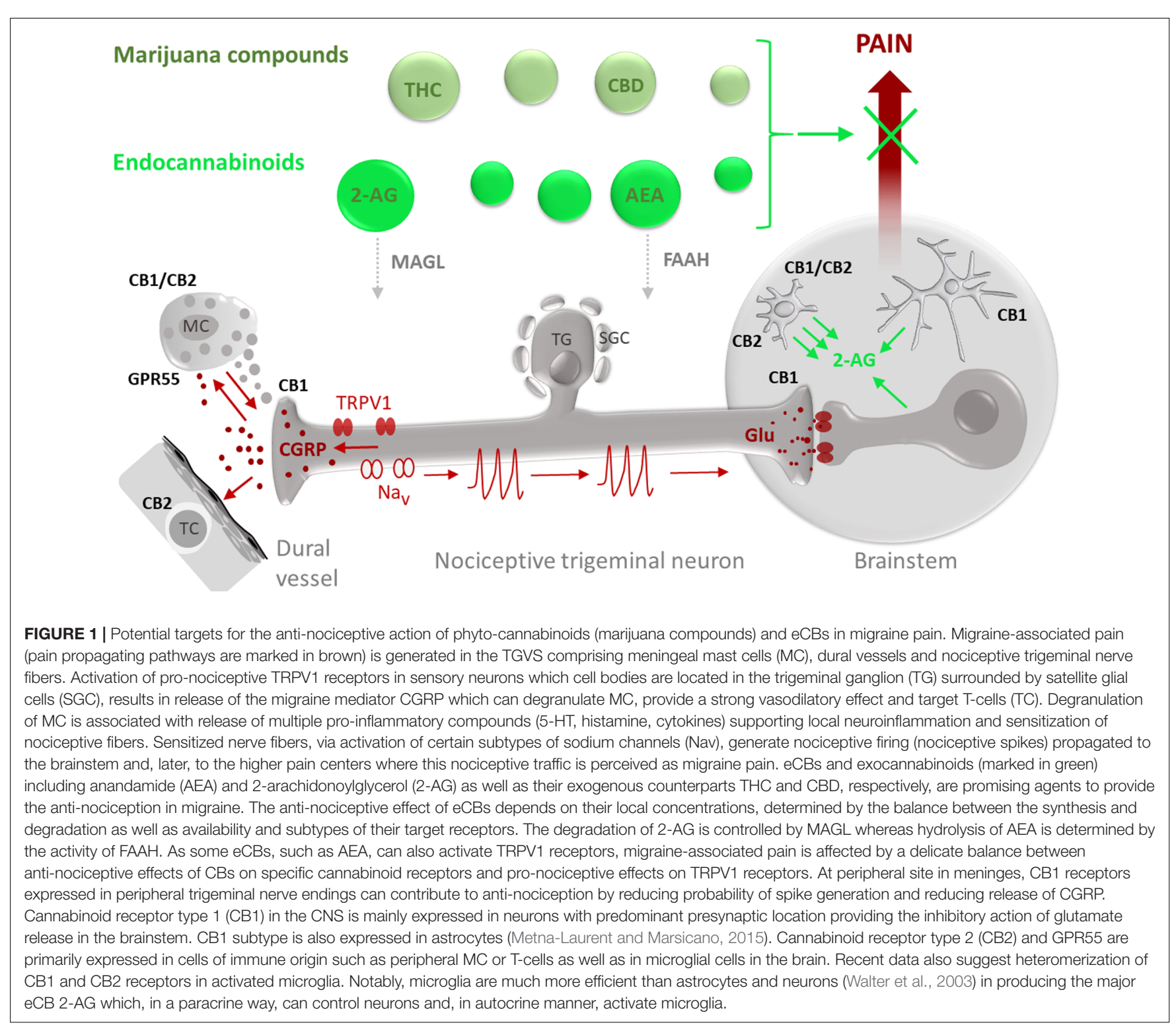

afferents in meninges (Kilinc et al., 2017; Figure 1). Notably, eCB operating via $\mathrm{CB} 1$ receptors can stabilize MC (Sugawara et al., 2012) and this effect also contributes to the anti-migraine action of these compounds. However, other data suggest a role for CB2 and the orphan receptor GPR55 in the stabilizing action of cannabinoids on mast cell HMC-1 line (Cantarella et al., 2011). It is yet to be studied using migraine models, but similar mast cell stabilizing effect in meninges could potentially contribute to the anti-migraine action of cannabinoids (Figure 1).

Cannabinoid effects on the CNS are mediated primarily by inhibitory CB1 receptors, located throughout CNS as well as in afferent neurons (Marco et al., 2012). Both within CNS and peripherally, eCBs act as retrograde messengers or synaptic modulators for their respective target cells (Gabral et al., 2015). Thus, one of the main functions of the eCB 2-AG, degraded by the enzyme monoacylglycerol lipase (MAGL, Aaltonen et al., 2016), is to serve as a mediator of retrograde signaling to downregulate neurotransmitter release (Smith et al., 2017). Unlike the selective presynaptic inhibitory effect of adenosine on excitatory glutamatergic terminals (Safiulina et al., 2005), activation of CB1 receptor by eCB inhibits the release from presynaptic terminals of both inhibitory and excitatory neurotransmitters (Gabrielli et al., 2015; Iseger and Bossong, 2015).

$\mathrm{CB} 2$ receptor, being, like $\mathrm{CB} 1$ receptor, highly sensitive to $2-\mathrm{AG}$, possesses an individual set of expression patterns and characteristic functions. Thus, CB2 expression is higher in peripheral organs than in the CNS and is mostly restricted to the immune system cells including B and T lymphocytes (Figure 1). Endocannabinoid system contributes to both innate and adaptive immune responses, functioning as a preventative force against the onset of pro-inflammatory responses (Nagarkatti et al., 2009; Oláh et al., 2017). CB2 receptors are primarily responsible for exerting immunosuppressive effects in the periphery. During 
an inflammatory reaction, which is expected in most severe or chronic forms of migraine, more of $\mathrm{CB} 2$ receptors are made available for activation (Gabral et al., 2015). In our recent study, familial migraine was found to be associated with enhanced concentrations of key inflammatory cytokines detected in blood (Khaiboullina et al., 2017). Thus, cannabinoids may act by correcting the dysregulation of cytokine production (Nagarkatti et al., 2009). Taken together, these studies suggest that the less explored $\mathrm{CB} 2$ receptors possessing the anti-inflammatory potential (Gabral et al., 2015) represent a promising target to counteract migraine (Scherma et al., 2016).

Besides their independent functions, CB1 and CB2 receptors have been shown to work together by forming hetero-receptor complexes (Callen et al., 2012). This type of receptor-receptor interaction has been shown recently for brain-residing immune cells such as microglia. Thus, it has been recently shown that, alongside $\mathrm{CB} 2$ receptors, the $\mathrm{CB} 1-\mathrm{CB} 2$ heteroreceptor complexes are expressed in microglia (Smith et al., 2017; Navarro et al., 2018; Figure 1). Microglia could play a part in the pathogenesis of migraine with aura, since the cortical spreading depression (CSD) associated with this type of migraine effectively activates microglia (Shibata and Suzuki, 2017). CSD also releases ATP (Karatas et al., 2013), which is a major driver of microglia, promoting release of the eCB 2-AG (Walter et al., 2003). Notably, the ability of microglia to secrete $2-\mathrm{AG}$ is about 20-times higher than that of astrocytes and neurons (Walter et al., 2003). In view of the recent data, this link appears to be even more intriguing as microglia are essential for initiation of CSD (Pusic et al., 2014). Interestingly, this positive feedback loop could be disrupted by agonists of $\mathrm{CB} 1$ (but not of $\mathrm{CB} 2$ receptors), which block CSD (Kazemi et al., 2012). Consistent with growing interest to the medications targeting receptor heteromers, a study using the bivalent CB1 antagonist specifically affecting dimerized CB1 receptors, showed pain-alleviating effects (Zhang et al., 2010). Overall, di- and oligomerization of GPCRs within CNS represent an attractive therapeutic target in pain conditions (Borroto-Escuela et al., 2013; Fuxe and BorrotoEscuela, 2015).

In peripheral migraine mechanisms, activation of TRPV1 receptor, a non-selective cation channel expressed in trigeminal nociceptors, leads to massive CGRP release (Kageneck et al., 2014; Figure 1). Our and other studies indicate an important contribution of TRPV1 receptors to migraine pathology (Zakharov et al., 2015; Dux et al., 2016). Stimulation of dural sensory nerves by capsaicin was found to cause vasodilation modulated by CGRP via TRPV1 receptor (Dux et al., 2016). As the TRPV1 channels can also bind eCB AEA (Chakrabarti et al., 2015), this may result in unwanted pro-nociceptive action of cannabinoids, causing neuroinflammation in meninges. This complexity may explain why increased doses of cannabinoids diminished their analgesic effect (Kandasamy et al., 2018). It further creates an incentive for development of new synthetic CBs with minimal activity on TRPV1 receptors, or specific MAGL inhibitors, which, apart from triggering the accumulation of anti-nociceptive 2-AG, can decrease the level of the pro-nociceptive arachidonic acid (AA) and reduce pain (Aaltonen et al., 2016). MAGL inhibitors may also reduce the pro-nociceptive downstream products of AA such as endovanilloids, agonists of TRPV1 receptors (Hwang et al., 2000). Interestingly, the inhibition of fatty acid amide hydrolase (FAAH) degrading AEA is also anti-nociceptive in migraine models (Greco et al., 2015).

\section{CLINICAL ENDOCANNABINOID DEFICIENCY (CECD) HYPOTHESIS}

Endocannabinoid system's role in homeostatic upkeep highlights the importance of this system in maintaining overall health. Disruptions in supply or functionality of eCB ligands have been connected to numerous mental state disturbances and, particularly, to migraine. Migraine, along with comorbid conditions such as fibromyalgia and irritable bowel syndrome, share symptomatic commonalities of hyperalgesia as well as treatment resistance, likely stemming from common pathophysiological phenomenon: CECD. The CECD hypothesis suggests a correlation between deficient levels of eCB and pain (Russo, 2016).

Since the initial proposal of the CEDC in 2001, the importance of maintaining regular $\mathrm{eCB}$ levels was shown in a study comparing $\mathrm{CB} 1$ - and $\mathrm{CB} 2-\mathrm{KO}$ mice that experienced inflammation, to mice lacking FAAH (and thus having elevated AEA) with reduced inflammation responses (Oláh et al., 2017). The lowered inhibitory activity of eCS in migraine, possibly due to reduced $\mathrm{CB} 1$ and $\mathrm{CB} 2$ receptor expression, serves as an assertion for the compensatory therapy with exogenous cannabinoids. According to the CECD hypothesis, treatment of migraine using exogenous cannabinoids could be achieved with low doses due to predisposition for elevated neuronal excitability. The CECD-causing deficiencies can appear for congenital reasons, or can be acquired.

\section{PRO AND CONTRA OF CANNABINOIDS IN MIGRAINE TREATMENT}

There is a long history of using cannabinoids for effective treatment of pain conditions. Due to their long-standing status of out-lawed substances (Baron, 2015), it is worth taking a look at the arguments still standing in the way of legalization. Overall, targeting ECS with peripherally acting drugs is a promising strategy for development of safe migraine treatments. However, there are still many insufficiently explored issues that may be detrimental for this seemingly harmless treatment.

Regularly experiencing chronic migraine pain can have adverse impacts on social relationships and job status which can lead into psychological distress (Ramsden et al., 2015). As it stands, the first 'pro' is that the treatment with pCB can acutely alleviate the resulting stress, in addition to tackling the initial cause by pain reduction.

In a study of the cannabis use for self-medication in Germany, Austria and Switzerland, $10.2 \%$ of patients reported using it 
for migraine headache symptoms (Kandasamy et al., 2018). Another group found that the self-treatment outcome was highly variable, with low doses tending to alleviate migraine while higher doses even triggering headaches (Lu and Anderson, 2017). These findings call for creating a highly specific prescription for individual patients, which would be required for safe and successful treatment plan.

One of the main problems arising from the long-term usage of cannabis is the physical reliance on the pCBs, mainly THC. Moreover, there is evidence that patients can develop tolerance for pCBs (Kandasamy et al., 2018). Carelessly establishing a reliance on any form of medication may carry more ill effects on the patient's mentality, and may even lead to weakening or loss of pain relief.

A crucial point when considering cannabinoid treatment is that smoking marijuana is the most common method of pCB self-administration. When self-administering pCBs via smoking, the relief seekers often use marijuana mixed with tobacco leaves. In view of the recently established crosstalk between nicotinic cholinergic and ECS (Scherma et al., 2016), the nicotinic cholinergic system has been proposed as a molecular target for treating cannabis dependence (Scherma et al., 2016). Particularly interesting is the ability of the endogenous nicotinic antagonist kynurenic acid to counteract the addictive effects of CBs (Justinova et al., 2013). Notably, new derivatives of kynurenic acid were suggested recently as promising medicines for migraine (Greco et al., 2017) opening a new perspective for combined $\mathrm{CB}+$ antinicotinic therapy of this disorder. Interestingly, the main migraine mediator CGRP can reduce the activity of nicotinic receptors (Giniatullin et al., 1999), suggesting that the migraines associated with enhanced level of endogenous CGRP are 'pre-conditioned' to respond better to $\mathrm{CB}$ treatments.

Cannabidiol (CBD), the second most prevalent pCB, should also be explored in relation to migraine treatment. Unlike THC with its characteristic CB1 receptor affinity, CBD does not have intoxicating and psychoactive effects linked with $\mathrm{CB} 1$ receptor activation. Yet, CBD possesses anxiolytic (anxiety-reducing) and antipsychotic properties that have been suggested to be inflicted via interactions with TRPV1 and non-endocannabinoid GPR55 receptor (Bih et al., 2015). Recently, the US Food and Drug Administration (FDA) accepted an application for Epidiolex ${ }^{\circledR}$ (active agent $\mathrm{CBD}$ ) in treatment of seizures prominent in Lennox-Gastaut syndrome (LGS) and Dravet syndrome (GW Pharmaceuticals, 2017). This stands as an important milestone paving the way for possible repurposing of this CBD-based drug for

\section{REFERENCES}

Aaltonen, N., Kedzierska, E., Orzelska-Górka, J., Lehtonen, M., NaviaPaldanius, D., Jakupovic, H., et al. (2016). In Vivo characterization of the ultrapotent monoacylglycerol lipase inhibitor $\{4$-[bis-(benzo[d][1,3]dioxol5-yl)methyl]-piperidin-1-yl\}(1H-1,2,4-triazol-1-yl)methanone (JJKK-048). J. Pharmacol. Exp. Ther. 359, 62-72. doi: 10.1124/jpet.116.233114 treating migraine, as well as other related neurological conditions.

\section{CONCLUSION}

In summary, cannabinoids - due to their anticonvulsive, analgesic, antiemetic, and anti-inflammatory effects - present a promising class of compounds for both acute and prophylactic treatment of migraine pain. In view of the rapidly unfolding changes in the legal status of cannabis, research on (endo)cannabinoids has become pertinent once again. Formal approval of a cannabinoid-based drug for other pathologies opens a possibility for repurposing these agents also to treat migraine. The abundance of $\mathrm{CB} 1$ receptors in the brain makes them an attractive target for treatment of migraine via blocking not only peripheral but also the central nociceptive traffic and reducing the pathologically enhanced cortical excitability predisposing to CSD. CB2 receptors in immune cells can be targeted to reduce the inflammatory component associated with severe forms of migraine. Exogenous compounds lacking the unwanted peripheral pro-nociceptive component or eCBs generated via inhibited degradation pathways and combined with other supportive agents are most desirable for this aim. Moreover, primary stratification of patients to identify and predict the effectiveness of cannabinoid treatment can greatly improve the efficiency of this approach.

\section{AUTHOR CONTRIBUTIONS}

PL, LK, and RG wrote the article and designed the figure.

\section{FUNDING}

RG was supported by the Finnish Academy (grant 277442) and by the program of competitive growth of Kazan Federal University and the subsidy $(6.2313 .2017 / 4.6)$ allocated to Kazan Federal University for the state assignment in the sphere of scientific activities.

\section{ACKNOWLEDGMENTS}

The authors are grateful to Jarmo Laitinen and Juha Savinainen for carefully reading the MS.

Aizpurua-Olaizola, O., Elezgarai, I., Rico-Barrio, I., Zarandona, I., Etxebarria, N., and Usobiaga, A. (2017). Targeting the endocannabinoid system: future therapeutic strategies. Drug Discov. Today 22, 105-110. doi: 10.1016/j.drudis. 2016.08.005

Akerman, S., Holland, P. R., and Goadsby, P. J. (2007). Cannabinoid (CB1) receptor activation inhibits trigeminovascular neurons. J. Pharmacol. Exp. Ther. 320, 64-71. doi: 10.1124/jpet.106.106971 
Akerman, S., Kaube, H., and Goadsby, P. J. (2003). Anandamide is able to inhibit trigeminal neurons using an in vivo model of trigeminovascular-mediated nociception. J. Pharmacol. Exp. Ther. 309, 56-63. doi: 10.1124/jpet.103.05 9808

Baron, E. P. (2015). Comprehensive review of medicinal marijuana, cannabinoids, and therapeutic implications in medicine and headache: what a long strange trip it's been. Headache 55, 885-916. doi: 10.1111/head.1 2570

Bih, C. I., Chen, T., Nunn, A. V. W., Bazelot, M., Dallas, M., and Whalley, J. B. (2015). Molecular targets of cannabidiol in neurological disorders. Neurotherapeutics 12, 699-730. doi: 10.1007/s13311-015-0377-3

Borroto-Escuela, D. O., Romero-Fernandez, W., Rivera, A., Van Craenenbroeck, K., Tarakanov, A. O., Agnati, L. F., et al. (2013). On the g-protein-coupled receptor heteromers and their allosteric receptorreceptor interactions in the central nervous system: focus on their role in pain modulation. Evid. Based Complement. Alternat. Med. 2013:563716. doi: $10.1155 / 2013 / 563716$

Burstein, R., Noseda, R., and Borsook, D. (2015). Migraine: multiple processes, complex pathophysiology. J. Neurosci. 35, 6619-6629. doi: 10.1523/JNEUROSCI.0373-15.2015

Callen, L., Moreno, E., Barroso-Chinea, P., Moreno-Delgado, D., Cortés, A., and Mallol, J. (2012). Cannabinoid receptors CB1 and CB2 form functional heteromers in brain. J. Biol. Chem. 287, 20851-20865. doi: 10.1074/jbc.M111. 335273

Cantarella, G., Scollo, M., Lempereur, L., Saccani-Jotti, G., Basile, F., and Bernardini, R. (2011). Endocannabinoids inhibit release of nerve growth factor by inflammation-activated mast cells. Biochem. Pharmacol. 82, 380-388. doi: 10.1016/j.bcp.2011.05.004

Chakrabarti, B., Persico, A., Battista, N., and Maccarrone, M. (2015). Endocannabinoid signaling in autism. Neurotherapeutics 12, 837-847. doi: 10.1007/s13311-015-0371-9

Dux, M., Deák, É., Tassi, N., Sántha, P., and Jancsó, G. (2016). Endovanilloids are potential activators of the trigeminovascular nocisensor complex. J. Headache Pain 17:53. doi: 10.1186/s10194-016-0644-7

Eroli, F., Loonen, I. C. M., van den Maagdenberg, A. M. J. M., Tolner, E. A., and Nistri, A. (2017). Differential neuromodulatory role of endocannabinoids in the rodent trigeminal sensory ganglion and cerebral cortex relevant to pain processing. Neuropharmacology 131, 39-50. doi: 10.1016/j.neuropharm.2017. 12.013

Fonseca, B. M., Costa, M. A., Almada, M., Correia-da-Silva, G., and Teixera, N. A. (2013). Endogenous cannabinoids revisited: a biochemistry perspective. Prostaglandins Other Lipid Mediat. 10, 13-30. doi: 10.1016/j.prostaglandins. 2013.02.002

Fuxe, K., and Borroto-Escuela, D. O. (2015). Heteroreceptor complexes and their allosteric receptor-receptor interactions as a novel biological principle for integration of communication in the CNS: targets for drug development. Neuropsychopharmacology 41, 380-382. doi: 10.1038/npp.2015.244

Gabral, G. A., Rogers, T. J., and Lichtman, A. H. (2015). Turning over a new leaf: cannabinoid and endocannabinoid modulation of immune function. J. Neuroimmune Pharmacol. 10, 193-203. doi: 10.1007/s11481-015-9 $615-\mathrm{z}$

Gabrielli, M., Battista, N., Riganti, L., Prada, I., Antonucci, F., Cantone, L., et al. (2015). Active endocannabinoids are secreted on extracellular membrane vesicles. EMBO Rep. 16, 213-220. doi: 10.15252/embr.201439668

Giniatullin, R., Di Angelantonio, S., Marchetti, C., Sokolova, E., Khiroug, L., and Nistri, A. (1999). Calcitonin gene-related peptide rapidly downregulates nicotinic receptor function and slowly raises intracellular $\mathrm{Ca} 2+$ in rat chromaffin cells in vitro. J. Neurosci. 19, 2945-2953. doi: 10.1523/JNEUROSCI. 19-08-02945.1999

Giniatullin, R., Nistri, A., and Fabbretti, E. (2008). Molecular mechanisms of sensitization of pain-transducing $\mathrm{P} 2 \mathrm{X} 3$ receptors by the migraine mediators CGRP and NGF. Mol. Neurobiol. 37, 83-90. doi: 10.1007/s12035-008-8 $020-5$

Gouveia-Figueira, S., Goldin, K., Hashemian, S. A., Lindberg, A., Persson, M., Nording, M. L., et al. (2017). Plasma levels of the endocannabinoid anandamide, related $\mathrm{N}$-acylethanolamines and linoleic acid-derived oxylipins in patients with migraine. Prostaglandins Leukot. Essent. Fatty Acids 120, 15-24. doi: 10.1016/j.plefa.2017.04.005
Greco, R., Bandiera, T., Mangione, A. S., Demartini, C., Siani, F., Nappi, G., et al. (2015). Effects of peripheral FAAH blockade on NTG-induced hyperalgesiaevaluation of URB937 in an animal model of migraine. Cephalalgia 35, 10651076. doi: 10.1177/0333102414566862

Greco, R., Demartini, C., Zanaboni, A. M., Redavide, E., Pampalone, S., Toldi, J., et al. (2017). Effects of kynurenic acid analogue 1 (KYNA-A1) in nitroglycerininduced hyperalgesia: targets and anti-migraine mechanisms. Cephalalgia 37, 1272-1284. doi: 10.1177/0333102416678000

Hwang, S. W., Cho, H., Kwak, J., Lee, S. Y., Kang, C. J., Jung, J., et al. (2000). Direct activation of capsaicin receptors by products of lipoxygenases: endogenous capsaicin-like substances. Proc. Natl. Acad. Sci. U.S.A. 97, 6155-6160. doi: 10.1073/pnas.97.11.6155

ICHD-3 beta (2013). The international classification of headache disorders, 3rd edition (beta version). Cephalalgia 33, 629-808. doi: 10.1177/ 0333102413485658

Iseger, T. A., and Bossong, M. G. (2015). A systematic review of the antipsychotic properties of cannabidiol in humans. Schizophr. Res. 162, 153-161. doi: 10.1016/ j.schres.2015.01.033

Justinova, Z., Mascia, P., Wu, H. Q., Secci, M. E., Redhi, G. H., Panlilio, L. V., et al. (2013). Reducing cannabinoid abuse and preventing relapse by enhancing endogenous brain levels of kynurenic acid. Nat. Neurosci. 16, 1652-1661. doi: $10.1038 / \mathrm{nn} .3540$

Kageneck, C., Nixdorf-Bergweiler, B. E., Messlinger, K., and Fischer, M. J. (2014). Release of CGRP from mouse brainstem slices indicates central inhibitory effect of triptans and kynurenate. J. Headache Pain 15:7. doi: 10.1186/1129-237 7-15-7

Kandasamy, R., Dawson, C. T., Craft, R. M., and Morgan, M. M. (2018). Antimigraine effect of $\Delta 9$-tetrahydrocannabinol in the female rat. Eur. J. Pharmacol. 818, 271-277. doi: 10.1016/j.ejphar.2017.10.054

Karatas, H., Erdener, S. E., Gursoy-Ozdemir, Y., Lule, S., Eren-Koçak, E., Sen, Z. D., et al. (2013). Spreading depression triggers headache by activating neuronal Panx1 channels. Science 339, 1092-1095. doi: 10.1126/science.123 1897

Kazemi, H., Rahgozar, M., Speckmann, E. J., and Gorji, A. (2012). Effect of cannabinoid receptor activation on spreading depression. Iran. J. Basic Med. Sci. 15, 926-936.

Khaiboullina, S. F., Mendelevich, E. G., Shigapova, L. H., Shagimardanova, E., Gazizova, G., Nikitin, A., et al. (2017). Cerebellar atrophy and changes in cytokines associated with the CACNA1A R583Q mutation in a Russian familial hemiplegic migraine type 1 family. Front. Cell. Neurosci. 11:263. doi: 10.3389/ fncel.2017.00263

Kilinc, E., Guerrero-Toro, C., Zakharov, A., Vitale, C., Gubert-Olive, M., Koroleva, K., et al. (2017). Serotonergic mechanisms of trigeminal meningeal nociception: implications for migraine pain. Neuropharmacology 116, 160-173. doi: 10.1016/j.neuropharm.2016.12.024

Levy, D. (2010). Migraine pain and nociceptor activation-where do we stand? Headache 50, 909-916. doi: 10.1111/j.1526-4610.2010.01670.x

Lu, Y., and Anderson, H. D. (2017). Cannabinoid signaling in health and disease. Can. J. Physiol. Pharmacol. 95, 311-327. doi: 10.1139/cjpp-20160346

Maccarrone, M., Dainese, E., and Oddil, S. (2010). Intracellular trafficking of anandamide: new concepts for signaling. Trends Biochem. Sci. 35, 601-608. doi: 10.1016/j.tibs.2010.05.008

Marco, E. M., Romero-Zerbo, S. Y., Viveros, M. P., and Bermudez-Silva, F. J. (2012). The role of the endocannabinoid system in eating disorders: pharmacological implications. Behav. Pharmacol. 23, 526-536. doi: 10.1097/ FBP.0b013e328356c3c9

Marsicano, G., Goodenough, S., Monory, K., Hermann, H., Eder, M., Cannich, A., et al. (2003). CB1 cannabinoid receptors and on-demand defense against excitotoxicity. Science 302, 84-88. doi: 10.1126/science.1088208

Metna-Laurent, M., and Marsicano, G. (2015). Rising stars: modulation of brain functions by astroglial type-1 cannabinoid receptors. Glia 63, 353-364. doi: $10.1002 /$ glia.22773

Nagarkatti, P., Pandey, R., Rieder, S. A., Hedge, V. L., and Nagarkatti, M. (2009). Cannabinoids as novel anti-inflammatory drugs. Future Med. Chem. 1, 1333-1349. doi: 10.4155/fmc.09.93

Navarro, G., Borroto-Escuela, D., Angelats, E., Etayo, I., Reyes-Resina, I., Pulido-Salgado, M., et al. (2018). Receptor-heteromer mediated regulation 
of endocannabinoid signaling in activated microglia. Role of CB1 and CB2 receptors and relevance for Alzheimer's disease and levodopa-induced dyskinesia. Brain Behav. Immun. 67, 139-151. doi: 10.1016/j.bbi.2017. 08.015

Niyuhire, F., Varvel, S. A., Martin, B. R., and Lichtman, A. H. (2007). Exposure to marijuana smoke impairs memory retrieval in mice. J. Pharmacol. Exp. Ther. 322, 1067-1075. doi: 10.1124/jpet.107.119594

Oláh, A., Szekanecz, Z., and Bíró, T. (2017). Targeting cannabinoid signaling in the immune system: "High"-ly exciting questions, possibilities, and challenges. Front. Immunol. 8:1487. doi: 10.3389/fimmu.2017.01487

Parker, L. A., Rock, E. M., and Limebeer, C. L. (2011). Regulation of nausea and vomiting by cannabinoids. Br. J. Pharmacol. 163, 1411-1422. doi: 10.1111/j. 1476-5381.2010.01176.x

Pavlovic, J. M., Buse, D. C., Sollars, C. M., Haut, S., and Lipton, R. B. (2014). Trigger factors and premonitory features of migraine attacks: summary of studies. Headache 54, 1670-1679. doi: 10.1111/head.12468

GW Pharmaceuticals (2017). GW Pharmaceuticals Announces Acceptance of NDA Filing for Epidiolex (cannabidiol) in the Treatment of Lennox-Gastaut Syndrome and Dravet Syndrome. Available at: https://www.gwpharm.com/about-us/news/gw-pharmaceuticals-announcesacceptance-nda-filing-epidiolex\%C2\%AE-cannabidiol-treatment [accessed January 22, 2018].

Pietrobon, D., and Moskowitz, M. A. (2013). Pathophysiology of migraine. Annu. Rev. Physiol. 75, 365-391. doi: 10.1146/annurev-physiol-030212-183717

Preedy, V. R. (2017). Handbook of Cannabis and Related Pathologies: Biology, Pharmacology. Cambridge, MA: Academic Press, 278-287.

Pusic, K. M., Pusic, A. D., Kemme, J., and Kraig, R. P. (2014). Spreading depression requires microglia and is decreased by their M2a polarization from environmental enrichment. Glia 62, 1176-1194. doi: 10.1002/glia.2 2672

Ramsden, C. E., Faurot, K. R., Zamora, D., Palsson, O. S., MacIntosh, B. A., Gaylord, S., et al. (2015). Targeted alterations in dietary n-3 and n-6 fatty acids improve life functioning and reduce psychological distress among chronic headache patients: secondary analysis of a randomized trial. Pain 156, 587-596. doi: 10.1097/01.j.pain.0000460348.84965.47

Rosenberg, E. C., Tsien, R. W., Whalley, B. J., and Devinsky, O. (2015). Cannabinoids and Epilepsy. Neurotherapeutics 12, 747-768. doi: 10.1007/ s13311-015-0375-5

Russo, A. F. (2015). Calcitonin gene-related peptide (CGRP): new target for migraine. Annu. Rev. Pharmacol. Toxicol. 55, 533-552. doi: 10.1146/annurevpharmtox-010814-124701

Russo, E. B. (2016). Clinical endocannabinoid deficiency reconsidered: current research supports the theory in migraine, fibromyalgia, irritable bowel, and other treatment-resistant syndromes. Cannabis Cannabinoid Res. 1, 154-165. doi: 10.1089/can.2016.0009

Safiulina, V. F., Kasyanov, A. M., Giniatullin, R., and Cherubini, E. (2005). Adenosine down-regulates giant depolarizing potentials in the developing rat hippocampus by exerting a negative control on glutamatergic inputs. J. Neurophysiol. 94, 2797-2804. doi: 10.1152/jn.00445.2005
Scherma, M., Muntoni, A. L., Melis, M., Fattore, L., Fadda, P., Fratta, W., et al. (2016). Interactions between the endocannabinoid and nicotinic cholinergic systems: preclinical evidence and therapeutic perspectives. Psychopharmacology 233, 1765-1777. doi: 10.1007/s00213-015-4196-3

Shibata, M., and Suzuki, N. (2017). Exploring the role of microglia in cortical spreading depression in neurological disease. J. Cereb. Blood Flow Metab. 37, 1182-1191. doi: 10.1177/0271678X17690537

Smith, D. R., Stanley, C. M., Foss, T., Boles, R. G., and McKernan, K. (2017). Rare genetic variants in the endocannabinoid system genes CNR1 and DAGLA are associated with neurological phenotypes in humans. PLoS One 12:e0187926. doi: 10.1371/journal.pone.0187926

Sugawara, K., Bíró, T., Tsuruta, D., Tóth, B. I., Kromminga, A., Zákány, N., et al. (2012). Endocannabinoids limit excessive mast cell maturation and activation in human skin. J. Allergy Clin. Immunol. 129, 726.e8-738.e8. doi: 10.1016/j.jaci. 2011.11.009

Sugiura, T., Kobayashi, Y., Oka, S., and Waku, K. (2002). Biosynthesis and degradation of anandamide and 2-arachidonoylglycerol and their possible physiological significance. Prostaglandins Leukot. Essent. Fatty Acids 66, 173192. doi: 10.1054/plef.2001.0356

Villalón, C. M., and Olesen, J. (2009). The role of CGRP in the pathophysiology of migraine and efficacy of CGRP receptor antagonists as acute antimigraine drugs. Pharmacol. Ther. 124, 309-323. doi: 10.1016/j.pharmthera.2009. 09.003

Walter, L., Franklin, A., Witting, A., Wade, C., Xie, Y., Kunos, G., et al. (2003). Nonpsychotropic cannabinoid receptors regulate microglial cell migration. J. Neurosci. 15, 1398-1405. doi: 10.1523/JNEUROSCI.23-04-01398. 2003

Zakharov, A., Vitale, C., Kilinc, E., Koroleva, K., Fayuk, D., Shelukhina, I., et al. (2015). Hunting for origins of migraine pain: cluster analysis of spontaneous and capsaicin-induced firing in meningeal trigeminal nerve fibers. Front. Cell. Neurosci. 9:287. doi: 10.3389/fncel.2015.00287

Zhang, Y., Gilliam, A., Maitra, R., Damaj, M. I., Tajuba, J. M., Seltzman, H. H., et al. (2010). Synthesis and biological evaluation of bivalent ligands for the CB1 receptor. J. Med. Chem. 53, 7048-7060. doi: 10.1021/jm1006676

Conflict of Interest Statement: LK is a founder and stake-holder of the company Neurotar LTD.

The other authors declare that the research was conducted in the absence of any commercial or financial relationships that could be construed as a potential conflict of interest.

Copyright (c) 2018 Leimuranta, Khiroug and Giniatullin. This is an open-access article distributed under the terms of the Creative Commons Attribution License (CC BY). The use, distribution or reproduction in other forums is permitted, provided the original author(s) and the copyright owner are credited and that the original publication in this journal is cited, in accordance with accepted academic practice. No use, distribution or reproduction is permitted which does not comply with these terms. 